See discussions, stats, and author profiles for this publication at: https://www.researchgate.net/publication/328030427

\title{
Is Self-Compassion a Worthwhile Therapeutic Target for ICD-11 Complex PTSD (CPTSD)?
}

Article in Behavioural and Cognitive Psychotherapy · October 2018

DOI: $10.1017 /$ \$1352465818000577

CITATIONS

12 authors, including:

Thanos Karatzias

Edinburgh Napier University

153 PUBLICATIONS 2,303 CITATIONS

SEE PROFILE

Aoife Bradley

Newcastle University

9 PUBLICATIONS 93 CITATIONS

SEE PROFILE

Some of the authors of this publication are also working on these related projects:

Desmond ID diabetes research study View project

Project

3MDR RCT View project
532

3 Philip Hyland

181 PUBLICATIONS 2,242 CITATIONS

SEE PROFILE

Jonathan I Bisson

202 PUBLICATIONS 9,082 CITATIONS

SEE PROFILE 


\title{
Is Self-Compassion a Worthwhile Therapeutic Target for ICD-11 Complex PTSD (CPTSD)?
}

\author{
Thanos Karatzias \\ Edinburgh Napier University, School of Health and Social Care, Edinburgh, UK, and NHS Lothian, \\ Rivers Centre for Traumatic Stress, Edinburgh, UK \\ Philip Hyland \\ National College of Ireland, School of Business, Dublin, Ireland, and Centre for Global Health, Trinity \\ College Dublin, Dublin, Ireland
}

Aoife Bradley

Edinburgh Napier University, School of Health and Social Care, Edinburgh, UK

Claire Fyvie

NHS Lothian, Rivers Centre for Traumatic Stress, Edinburgh, UK

Katharine Logan

NHS Lothian, Rivers Centre for Traumatic Stress, Edinburgh, UK

Paula Easton

NHS Lothian, Rivers Centre for Traumatic Stress, Edinburgh, UK

Jackie Thomas

NHS Lothian, Rivers Centre for Traumatic Stress, Edinburgh, UK

Sarah Philips

NHS Lothian, Rivers Centre for Traumatic Stress, Edinburgh, UK

Correspondence to Professor Thanos Karatzias, Edinburgh Napier University, Sighthill Campus, Sighthill Court, Edinburgh EH11 4BN, UK. E-mail: t.karatzias@ napier.ac.uk 


\author{
Jonathan I. Bisson
}

Cardiff University, School of Medicine, Cardiff, UK

Neil P. Roberts

Psychology and Psychological Therapies Directorate, Cardiff and Vale University Health Board, Cardiff, UK, and Cardiff University, School of Medicine, Cardiff, UK

Marylene Cloitre

National Center for PTSD, Palo Alto Health Care System, Stanford University, Palo Alto, CA, USA

Mark Shevlin

Ulster University, School of Psychology, Derry, Northern Ireland

\begin{abstract}
Background: Two 'sibling' disorders have been proposed for the fourthcoming 11th version of the International Classification of Diseases (ICD-11): post-traumatic stress disorder (PTSD) and complex PTSD (CPTSD). Examining psychological factors that may be associated with CPTSD, such as self-compassion, is an important first step in its treatment that can inform consideration of which problems are most salient and what interventions are most relevant. Aims: We set out to investigate the association between self-compassion and the two factors of CPTSD: the PTSD factor (re-experiencing, avoidance, sense of threat) and the Disturbances in Self-Organization (DSO) factor (affect dysregulation, negative self-concept and disturbances in relationships). We hypothesized that self-compassion subscales would be negatively associated with both PTSD and DSO symptom clusters. Method: A predominantly female, clinical sample $(n=106)$ completed self-report scales to measure traumatic life events, ICD-11 CPTSD and self-compassion. Results: Significant negative associations were found between the CPTSD DSO clusters of symptoms and self-compassion subscales, but not for the PTSD ones. Specifically it was also found that self-judgement and common humanity significantly predicted hypoactive affect dysregulation whereas self-judgement and isolation significantly predicted negative self-concept. Conclusions: Our results indicate that self-compassion may be a useful treatment target for ICD-11 CPTSD, particularly for symptoms of negative selfconcept and affect dysregulation. Future research is required to investigate the efficacy and acceptability of interventions that have implicit foundations on compassion.
\end{abstract}

Keywords: self-compassion, psychological trauma, CPTSD, ICD-11

\title{
Introduction
}

'Third wave' cognitive behavioural therapies, such as acceptance and commitment therapy (ACT; Hayes et al., 1999), mindfulness-based cognitive therapy (MBCT; Segal et al., 2002), dialectical behavioural therapy (DBT; Linehan, 1993) and compassion-focused therapy (CFT; Gilbert, 2010) all focus on ameliorating psychological distress by changing the person's relationship with their problems. One way to achieve this is to develop a more 
compassionate attitude towards one's self (i.e. self-compassion). Neff (2003a, p. 87) defines self-compassion as 'being touched by and open to one's own suffering, not avoiding or disconnecting from it, generating the desire to alleviate one's suffering and to heal oneself with kindness. Self-compassion also involves offering non-judgmental understanding to one's pain, inadequacies and failures, so that one's experience is seen as part of the larger human experience'.

Self-compassion has been described as involving three distinct elements including (1) being kind and understanding toward oneself in times of difficulty, (2) being mindfully aware of painful thoughts and feelings to prevent over-identification, and (3) seeing one's struggles as part of a broader human experience rather than as a unique and isolating experience (Neff, 2011). Thus, self-compassion can reduce self-judgement, rumination, and feelings of isolation. Self-compassion can also act as an adaptive coping mechanism that increases resilience, positive cognitions and decreases avoidance-oriented coping (Allen and Leary, 2010; Krieger et al., 2013). For all these reasons, self-compassion has been identified as a worthwhile therapeutic target for anxiety, depression and stress (MacBeth and Gumley, 2012). A number of studies have highlighted the protective effects of self-compassion following exposure to stressors. For example, in a recent study, shame-prone individuals were randomized to describe a shameful event or reflect on the event with self-compassion. It was found that those instructed to adopt a self-compassionate position had less shame, rumination, and depressive symptoms relative to those who did not (Johnson and O'Brien, 2013).

The association between post-traumatic stress disorder (PTSD) symptoms and selfcompassion has also been explored in previous research. Thompson and Waltz (2008) found that in a sample of college students self-compassion was negatively correlated with symptoms of avoidance, but not re-experiencing or hyperarousal. Furthermore, Maheux and Price (2015) investigated the association between self-compassion and PTSD symptomatology in two general population samples. They found that self-compassion was negatively correlated with aggregated PTSD symptoms for both DSM-IV and DSM-5 descriptions of PTSD. Selfcompassion was correlated with avoidance symptoms for DSM-IV and with all symptom clusters for the DSM-5. There is, however, less research on the association between PTSD symptomatology and self-compassion in clinical samples, although existing studies report similar findings with the general population. In a sample of treatment-seeking women who were survivors of interpersonal violence with PTSD, Scoglio et al. (2015) reported a significant negative relationship between PTSD symptoms and self-compassion.

The forthcoming 11th version of the International Classification of Diseases (ICD-11), produced by the World Health Organization (WHO), will include two trauma-based disorders: PTSD and complex PTSD (CPTSD; Maercker et al., 2013). The description of PTSD in ICD-11 is simpler than that provided within the DSM-5. In contrast to the 20 symptoms spread across four symptom clusters in DSM-5, ICD-11 PTSD includes just six 'core' symptoms across three clusters, each of which is directly related to one's traumatic exposure: re-experiencing in the here and now (Re: two symptoms), avoidance (Av: two symptoms), and a sense of current threat (Th: two symptoms). Diagnosis of ICD-11 PTSD requires the presence of one symptom per cluster, plus evidence of functional impairment. CPTSD is a broader diagnosis that includes the core PTSD symptoms plus an additional set of symptoms that are collectively referred to as 'disturbances in self-organization' (DSO). These symptoms are intended to capture pervasive psychological disturbances associated with experiences that typically consist of chronic or multiple types of traumatic exposure. DSO symptoms are distributed across three clusters: 
affective dysregulation, both hyperactivation and hypoactivation, negative self-concept, and disturbances in relationships. A CPTSD diagnosis requires that the PTSD criteria be met in addition to endorsement of symptoms from each of the DSO clusters.

The association between self-compassion and ICD-11 PTSD and DSO symptoms has never been explored before. Given the inclusion of symptoms relating to dysregulated emotions, persistent negative evaluations of the self, and relational difficulties in the ICD-11 CPTSD symptom profile, it is plausible that self-compassion will correlate more strongly with these trauma reactions, compared with the 'core' trauma reactions captured in the ICD-11 PTSD symptom profile. Given the paucity of clinical interventions that currently exist for ICD11 CPTSD, identification of a unique psychological correlate of this disorder could have important implications for the treatment of CPTSD. In the present study, we aimed to explore the associations between the different facets of self-compassion and each of the ICD-11 PTSD and DSO symptom clusters using a newly developed measure of CPTSD symptomatology [i.e. the International Trauma Questionnaire (ITQ); Cloitre et al., 2017] amongst a clinical sample of traumatized persons. We hypothesized that self-compassion subscales would be negatively associated with both PTSD and DSO symptom clusters.

\section{Methods}

\section{Participants and procedures}

Participants were referred by general practitioners, psychiatrists or psychologists for psychological therapy at a National Health Service trauma centre in Scotland $(n=106)$. Data were collected during an initial assessment using standardized scales. A psychology assistant was present if participants had any questions regarding the scales. The sample was primarily female (93.4\%) and of British origin (91.3\%) with a mean age of 39.25 years $(S D=10.94$, range 19-62). Most participants had finished post-secondary education (56.6\%), were currently unemployed (58.1\%), and single (59.2\%).

\section{Measures}

Childhood trauma. The Childhood Trauma Questionnaire (CTQ; Bernstein and Fink, 1998) is a 28-item, self-report questionnaire, that assesses exposure to a range of different childhood traumas. The scale produces five subscales, each with five items: Emotional Abuse, Physical Abuse, Sexual Abuse, Emotional Neglect, and Physical Neglect. Items are responded to using a 5-point scale ranging from 'never true' (1) to 'very often true' (5) with regard to the endorsed frequency of the event, and the mean scores for each subscale were calculated. The measure demonstrated good internal consistency, test-retest reliability, and convergent validity (Bernstein and Fink, 1998). In the present study, high levels of internal consistency were found for each of the subscales, (emotional abuse $\alpha=.79$, physical abuse $\alpha=.80$, sexual abuse $\alpha=.97$, emotional neglect $\alpha=.90$, physical neglect, $\alpha=.69$ ) and for the total scale $(\alpha=.87)$.

Traumatic life events. The Life Events Checklist (LEC; Gray et al., 2004) is a 17-item, self-report measure designed to screen for potentially traumatic events in a respondent's lifetime. The LEC assesses lifetime exposure to 16 traumatic events (e.g. natural disaster, physical assault, life threatening illness/injury) and the 17th item,'Any other very stressful 
event/experience', can be used to indicate exposure to a trauma that was not listed. For each item, the respondent checks whether the event 'Happened to me' (1), 'Witnessed it happening to somebody else' (2), 'Learned about it happening to someone close to me' (3), 'Part of my job' (4), 'Not sure it applies' (5), or 'Does not apply to my experience' (6). In order to create a summed total to represent the number of different traumatic life events that have been experienced, the items were recoded into binary variables with 'Happened to me' and 'Witnessed it' responses being coded as ' 1 ' and all other responses coded as ' 0 '. This produced a single total cumulative index variable with possible scores ranging from 0 to 16; item 17 was not included as the nature of the trauma could not be identified.

ICD-11 CPTSD. The ITQ (version 1.2; Cloitre et al., 2015; formerly the 'ICD-11 Trauma Questionnaire') is a self-report measure of the ICD-11 diagnoses of PTSD and CPTSD following exposure to traumatic events as defined by the CTQ and LEC. Six items measure the PTSD symptoms (two items per symptom cluster); 16 items measure the DSO symptoms (five items measure 'affective dysregulation-hyperactivation', four items measure 'affective dysregulation-hypoactivation', four items measure 'negative self-concept', three items measure 'disturbances in relationship'); and six items measure functional impairment associated with both the PTSD and DSO symptoms. Individuals respond to each PTSD item in terms of how much they have been bothered by the symptom over the past month, and respond to each DSO item in terms of how they typically feel, think about themselves, and relate to others. All items are measured using a five-point Likert scale ranging from 0 ('not at all') to 4 ('extremely'). Symptom severity scores for each PTSD and DSO symptom are calculated by summing responses to each item and, in every case, higher scores indicate greater symptom severity. The psychometric properties of the ITQ have been validated in a number of studies, including within a larger clinical sample (see Karatzias et al., 2016, 2017). The internal reliability of the PTSD $(\alpha=.74)$ and DSO $(\alpha=.89)$ items in the current sample were satisfactory.

Self-compassion. The Self-Compassion Scale (SCS; Neff, 2003b) is a 26-item self-report instrument that assesses trait levels of self-compassion across six subscales. The SCS includes items that measure how often people respond to feelings of inadequacy or suffering with: self-kindness (e.g. 'I try to be loving towards myself when I'm feeling emotional pain'), selfjudgement (e.g. 'I'm disapproving and judgemental about my own flaws and inadequacies'), common humanity (e.g. 'I try to see my failings as part of the human condition'), isolation (e.g. 'When I fail at something that's important to me, I tend to feel alone in my failure'), mindfulness (e.g. 'When something upsets me I try to keep my emotions in balance'), and over-identification (e.g. 'When I'm feeling down I tend to obsess and fixate on everything that's wrong'). Responses on the SCS are captured using a 5-point scale ranging from 'almost never' to 'almost always', with higher scores representing greater self-compassion. The SCS has demonstrated good internal consistency ( $\alpha=.77$ to .81 across total subscales) as well as good test-retest reliability ( $\alpha=.85$ to .93 across total and subscales). The SCS has also demonstrated good discriminant validity (e.g. low to moderate associations with self-esteem measures such as the Self-determination Scale, $r=.43$ ) and construct validity (e.g. association with DEQ selfcriticism subscale, $r=.65$ ) (Neff, 2003b). In addition, several studies using diverse samples have supported the validity of the six-factor structure of the SCS (e.g. Arimitsu, 2014; Azizi et al., 2013; Castilho et al., 2015). In the present study, good levels of internal consistency were found for each of the subscales (self-kindness $\alpha=.72$, self-judgement $\alpha=.83$, common 
humanity $\alpha=.70$, isolation $\alpha=.75$, mindfulness, $\alpha=.66$, over identified, $\alpha=.71)$ and for the total scale $(\alpha=.88)$.

\section{Data analysis}

Data were analysed using the Statistical Package for Social Sciences (SPSS), version 23. Means and standard deviations were calculated for continuous variables and frequencies (\%) for categorical variables (Table 1). To test for multicollinearity, relationships between continuous variables were investigated by Pearson's correlations, and the Tolerance and VIF statistics. No bivariate correlation exceeded 0.70 , all Tolerance values exceeded .10 and no VIF values exceeded 10 (Tabachnick and Fidell, 2011), thus no variables were excluded from the regression analysis. Standard multiple regression analyses were used to determine the unique associations between each facet of self-compassion and the different symptom clusters of ICD-11 PTSD (Re, Av, Th) and CPTSD (AD, NSC, DR). Each model also included age and gender as covariates (see Table 2). If an overall model was statistically significant, the percentage of explained variance attributable to each predictor was calculated and these estimates are added in parentheses in Table 2.

\section{Results}

The mean number of traumatic life events was $6.99(S D=2.80)$, with only a small number $(1.9 \%)$ reporting exposure to a single traumatic event; a total of $70.8 \%$ of the sample reported experiencing between three and eight traumatic events. The most commonly reported traumatic experiences were physical assault (95.1\%), and emotional abuse during childhood (93.1\%). All participants reported experiencing at least one traumatic life event. Both childhood and adulthood traumatic experiences had occurred in $94.3 \%$ of the sample. No participants reported exposure to traumatic events only in childhood, while $1 \%$ experienced traumatic events only in adulthood. The majority of the current sample met probable diagnostic requirements for either ICD-11 PTSD or CPTSD (79.8\%). The taxonomic structure of the ICD-11 only permits a diagnosis of PTSD or CPTSD, but not both. Accordingly, more individuals satisfied the diagnostic criteria for CPTSD (62.5\%) than PTSD (17.3\%).

The bivariate correlations between all self-compassion and PTSD and DSO symptom clusters are reported in Table 1. None of the self-compassion subscales was significantly associated with the three PTSD symptom clusters. Each self-compassion subscale was significantly and negatively correlated with the hypoactivation ( $r$ values ranged from -.27 to -.43 ) and negative self-concept ( $r$ values ranged from -.20 to -.45) symptom clusters of CPTSD. Four self-compassion subscales (self-kindness, self-judgement, common humanity, and isolation) were significantly and negatively correlated with disturbances in relationships ( $r$ values ranged from -.21 to -.26 ) and the over-identification subscale was the only selfcompassion facet associated with the hyperactivation cluster $(r=-.25)$. This pattern of association suggests that self-compassion is uniquely associated with CPTSD symptoms.

The PTSD and DSO symptom cluster scores were regressed onto the six facets of selfcompassion, while also controlling for gender and age (see Table 2 for all multiple regression results). The proportions of variance explained in the re-experiencing $(5.2 \%)$, avoidance $(5.3 \%)$ and sense of threat $(0.5 \%)$ PTSD symptom clusters were all very low and non-significant. With respect to the DSO symptom clusters, the proportion of variance explained in the 'affective 
Table 1. Means, standard deviations and correlations for self-compassion subscales and ICD-11 ITQ constructs

\begin{tabular}{lllllllll}
\hline & Means $(S D)$ & $\begin{array}{l}\text { Re-experiencing } \\
(\mathrm{Re})\end{array}$ & $\begin{array}{l}\text { Avoidance } \\
(\mathrm{Av})\end{array}$ & $\begin{array}{l}\text { Sense of } \\
\text { Threat (Th) }\end{array}$ & $\begin{array}{l}\text { Hyperactivity } \\
(\mathrm{AD})\end{array}$ & $\begin{array}{l}\text { Hypoactivity } \\
(\mathrm{AD})\end{array}$ & $\begin{array}{l}\text { Negative self- } \\
\text { concept (NSC) }\end{array}$ & $\begin{array}{l}\text { Disturbances in } \\
\text { relationships (DR) }\end{array}$ \\
\hline Self-kindness & $8.39(4.29)$ & -.036 & .019 & -.025 & -.060 & $-.293^{* *}$ & $-.206^{*}$ & $-.227^{*}$ \\
Self-judgement & $7.25(3.63)$ & -.097 & .010 & -.156 & -.168 & $-.378^{* *}$ & $-.448^{* *}$ & $-.258^{* *}$ \\
$\quad \begin{array}{l}\text { Common } \\
\quad \text { Humanity }\end{array}$ & $9.53(4.61)$ & -.176 & .025 & -.132 & -.149 & $-.413^{* *}$ & $-.199^{*}$ & $-.254^{*}$ \\
$\quad$ & & & & & & & \\
Isolation & $6.95(3.58)$ & -.173 & .060 & -.064 & -.144 & $-.431^{* *}$ & $-.346^{* *}$ & $-.212^{*}$ \\
Mindfulness & $9.83(4.38)$ & -.102 & .052 & -.038 & -.047 & $-.354^{* *}$ & $-.198^{*}$ & -.132 \\
Over-identified & $7.47(3.66)$ & -.034 & .058 & -.072 & $-.248^{*}$ & $-.268^{* *}$ & $-.259^{* *}$ & -.144 \\
Means $(S D)$ & & $5.30(2.36)$ & $5.88(1.68)$ & $6.02(2.11)$ & $12.19(4.21)$ & $10.79(3.81)$ & $12.36(4.10)$ & $8.93(2.67)$ \\
\hline
\end{tabular}

${ }^{* *} p<0.01,{ }^{*} p<0.05$ 
Table 2. Predicting ICD-11 PTSD and DSO constructs from self-compassion subscales

\begin{tabular}{|c|c|c|c|c|c|c|c|}
\hline \multicolumn{4}{|c|}{ PTSD } & \multicolumn{4}{|c|}{ DSO } \\
\hline & $\begin{array}{l}\text { Re-experiencing } \\
(\mathrm{Re})\end{array}$ & $\begin{array}{l}\text { Avoidance } \\
\text { (Av) }\end{array}$ & $\begin{array}{l}\text { Sense of } \\
\text { Threat (Th) }\end{array}$ & $\begin{array}{l}\text { Hyperactivation } \\
\text { (AD-Hyper) }\end{array}$ & $\begin{array}{l}\text { Hypoactivation } \\
\text { (AD-Hypo) }\end{array}$ & $\begin{array}{l}\text { Negative self- } \\
\text { concept (NSC) }\end{array}$ & $\begin{array}{l}\text { Disturbances in } \\
\text { relationships (DR) }\end{array}$ \\
\hline Predictors & $\beta$ & $\beta$ & $\beta$ & $\beta$ & $\beta$ & $\beta$ & $\beta$ \\
\hline Age & -0.04 & 0.11 & -0.01 & -0.07 & 0.02 & -0.06 & -0.00 \\
\hline Gender & $-0.21^{*}$ & 0.11 & -0.13 & 0.05 & 0.14 & 0.15 & -0.05 \\
\hline Self-kindness & 0.18 & -0.00 & 0.13 & 0.04 & 0.20 & 0.17 & -0.00 \\
\hline Self-judgement & -0.20 & -0.09 & -0.21 & -0.06 & $-0.35(4.2 \%)^{*}$ & $-0.62(9.8 \%)^{* * *}$ & -0.21 \\
\hline Common Humanity & -0.25 & -0.07 & -0.14 & -0.09 & $-0.29(4.9 \%)^{*}$ & -0.02 & -0.15 \\
\hline Isolation & -0.14 & 0.06 & 0.03 & -0.09 & -0.29 & $-0.36(4.1 \%)^{*}$ & -0.05 \\
\hline Mindfulness & -0.10 & 0.11 & 0.04 & 0.19 & 0.01 & 0.11 & 0.12 \\
\hline Over-identified & 0.20 & 0.06 & 0.08 & -0.26 & 0.23 & 0.21 & 0.08 \\
\hline $\operatorname{Adj} R^{2}$ & .052 & -.053 & -.005 & .049 & .211 & .292 & .038 \\
\hline$F$ & 1.69 & 0.37 & 0.94 & 1.63 & $4.28^{* * *}$ & $4.84^{* * *}$ & 1.48 \\
\hline
\end{tabular}

${ }^{* * *} p<.001,{ }^{* *} p=.01,{ }^{*} p<.05$. 
dysregulation-hyperactivity' (4.9\%) and 'disturbances in relationships' (3.8\%) symptoms were also low and non-significant. However, the self-compassion model explained 29.2\% of variance in 'negative self-concept' symptoms $\left(R^{2}=.292 ; F(8,94)=4.84, p<.001\right)$, and $21.1 \%$ of variance in 'affective dysregulation-hypoactivity' symptoms $\left(R^{2}=.211 ; F(8,90)=4.28, p<\right.$ $.001)$.

The 'affective dysregulation-hypoactivity' CPTSD symptom cluster was uniquely associated with two facets of self-compassion: self-judgement $[\beta=-.35$ (95\% CI $=-.67$ to -.03$), p<.05]$ and common humanity $(\beta=-.29 ; 95 \% \mathrm{CI}-.54$ to $-.05 ; p<.05)$. The 'negative self-concept' CPTSD symptom cluster was also uniquely associated with two facets of self-compassion: self-judgement $(\beta=-.62 ; 95 \% \mathrm{CI}-.95$ to $-.29 ; p<.001)$ and isolation ( $\beta=-.36 ; 95 \% \mathrm{CI}-.67$ to $-.06 ; p<.05)$. Overall, the results of the multiple regression analyses indicate that self-compassion is associated with CPTSD symptomatology but not PTSD symptomatology.

\section{Discussion}

We set out to investigate if self-compassion is uniquely associated with ICD-11 CPTSD symptom clusters so that we may identify a unique psychological correlate of this newly proposed diagnostic entity. Identifying psychological factors that are independently associated with CPTSD is an important first step in its treatment as these findings can inform consideration of which problems are most salient and what interventions are most relevant. The bivariate correlation results initially indicated that self-compassion was uniquely associated with specific CPTSD symptomatology including affect dysregulation (particularly the hypoactivity component of this symptom cluster), negative self-concept, and disturbed relationships. The unique association between self-compassion and specific CPTSD symptom clusters was further evidenced by the results of the regression analyses. These results revealed that self-judgement and common humanity significantly predicted hypoactive affect dysregulation symptoms, and that self-judgement and isolation significantly predicted negative self-concept symptoms. These results indicate that self-compassion may be a particularly important construct in predicting DSO symptoms compared with PTSD symptoms, particularly for symptoms of negative self-concept and affect dysregulation.

Negative self-concept symptoms are central to CPTSD and are defined in terms of persistent beliefs about oneself as diminished, defeated or worthless, and are accompanied by deep and pervasive feelings of shame, guilt or failure (Maercker et al., 2013). Such beliefs and schemas can make individuals very resistant to standard clinical interventions and it has been argued that such patterns of thinking might be more responsive to compassion-focused interventions (Gilbert and Irons, 2014). Our results support these claims and indicate that compassionfocused therapy might be a useful tool to address pervasive beliefs and schemas related to negative self-concept in trauma-exposed people.

On the basis of previous findings (e.g. Maheux and Price, 2015), it may be considered surprising that self-compassion was not found to be significantly associated with the ICD-11 PTSD symptom clusters. However, previous studies that have reported associations between self-compassion and PTSD were all derived from DSM-based assessments of PTSD. It may be that the association between self-compassion and PTSD symptoms, even including avoidance symptoms, may be mediated by disturbances in self-organization (DSO) symptoms which were not measured in previous studies. In addition, it is important to note that the DSM-IV 
and DSM-5 PTSD symptom profiles include 11 and 14 additional symptoms, respectively, compared with the ICD-11 PTSD symptom profile. Many of the symptoms that comprise the DSM-based models of PTSD are actually included within the ICD-11 description of CPTSD (e.g. irritability, emotional responsivity, emotional numbing, dissociative experiences, negative self-evaluations, and relational difficulties). As such, current findings may be considered to add meaningfully to the existing literature regarding the association between self-compassion and PTSD. By adopting the ICD-11's framework for trauma-related psychopathology, we were able to identify that at least in this clinical sample, self-compassion is not meaningfully associated with symptoms that are directly tied to one's traumatic exposure (e.g. re-experiencing, avoidance, hyperarousal), but are meaningfully associated with the symptoms that reflect disturbances in one's self-organization, especially relating to emotional hypoactivity, and negative self-concept. By more clearly delineating the relationship between self-compassion and trauma symptoms that was afforded through the use of the ICD-11 framework, the current findings can inform clinicians as to the possible usefulness of compassion-focused interventions when working with trauma-exposed individuals.

Compassion-focused strategies are already incorporated in a number of interventions. In mindfulness-based stress reduction (MBSR), for example, loving-kindness is introduced during an all-day meditation (Kabat-Zinn, 1990) and it has been suggested that this programme may be helpful in reducing anger in survivors of childhood sexual abuse (Bowman, 2005). Furthermore, an implicit foundation of compassion underlies a number of contextual behavioural therapies such as acceptance and commitment therapy (ACT; Hayes et al., 1999). Building on the work of Paul Gilbert (e.g. Gilbert and Irons, 2014), Deborah Lee (e.g. Lee and James, 2012; Lee, 2016) has recently proposed a model of intervention for PTSD based on compassion-focused therapy, which may be particularly beneficial for CPTSD. While current findings suggest that inclusion of compassion-focused interventions alongside traditional trauma-focused treatments may be beneficial for the treatment of CPTSD, the effectiveness of such interventions has not been explored as yet and these recommendations should be interpreted cautiously. There is clearly a need for further research on the effectiveness of various interventions for CPTSD that target both the 'core' PTSD symptoms, and the more psychologically pervasive DSO symptoms. There is also a need for dismantling studies to tease out the relative efficacy of different active treatment components for CPTSD.

Although our results indicate that strategies which improve self-compassion may potentially reduce DSO symptoms, the precise mechanisms by which the treatment effects of selfcompassion occur remain unknown and should be explored further in future experimental research. It has been argued that self-compassion can increase positive cognitions and decrease avoidance through increased mindfulness, defined as non-judgemental awareness of the present moment. Recent interventions for the treatment of PTSD that have used mindfulness techniques have demonstrated positive findings (Dutton et al., 2013; Kearney et al., 2012), which might be attributed to increased self-compassion. However, these interventions have never been tested in CPTSD and therefore future work is necessary to determine the extent and the mechanisms that self-compassion strategies might improve CPTSD symptoms.

There are several limitations in the current study. Analyses were based on correlations and it was not possible to investigate the direction in relationship between observed findings. The analyses were also based on a small clinical sample with severe traumatic symptomatology meaning that there was an increased likelihood of Type 2 errors occurring. Furthermore, the predominantly female composition of the sample and high level of exposure to childhood 
trauma limits the generalizability of findings to the wider trauma population. In addition, selfreport assessments rather than clinician-administered interviews were employed in the present study. It is possible that the self-report nature of the data may have biased results, and replication with clinician-administered measures would be beneficial to confirm current findings. Finally, there is content overlap in some variables (e.g. self-judgement and negative self-concept) potentially inflating the magnitude of association. However, there are some differences that suggest that these are tapping into different types of self-evaluation. The SCS asks participants to respond to the items based on 'How I typically act towards myself in difficult times', and uses a 'Never' to 'Always' response format. In contrast, the ITQ asks respondents 'The questions refer to ways you typically feel, ways you typically think about yourself and ways you typically relate to others' as a result of having experienced a traumatic stressor using a 'Not at all' to 'Extremely' response format. Furthermore, the magnitude of associations between SCS and ITQ reported in Table 1 are very reassuring and they are not denoting significant overlap between these two scales. Notwithstanding its limitations, this is the first study to explore the association between self-compassion and ICD-11 CPTSD and our results suggest that cultivating a self-compassion attitude during therapy may be useful in the treatment of CPTSD and particularly for symptoms of negative self-concept and affect dysregulation.

\section{Acknowledgements: None.}

Conflicts of interest: The authors have no conflicts of interest with respect to this publication.

Ethical statement: This work has been reviewed by the South East Scotland Research Ethics Service (NR/1502AB7) and ethics approval was not required because data were collected as part of usual care.

Funding: This research received no specific grant from any funding agency, commercial or not-for-profit sectors.

\section{References}

Allen, A. B. and Leary, M. R. (2010). Self-compassion, stress, and coping. Social and Personality Psychology Compass, 4, 107-118.

Arimitsu, K. (2014). Development and validation of the Japanese version of the Self-Compassion Scale. The Japanese Journal of Psychology, 85, 50-59.

Azizi, A., Mohammadkhani, P., Lotfi, S. and Bahramkhani, M. (2013). The validity and reliability of the Iranian version of the Self-Compassion Scale. Iranian Journal of Clinical Psychology, 2, 17-23.

Bernstein, D. P. and Fink, L. (1998). Childhood Trauma Questionnaire: A retrospective self-report manual. San Antonio, TX: The Psychological Corporation.

Bowman, K. (2005). Commentary of 'Loving-kindness meditation for chronic low back pain'. Journal of Holistic Nursing, 23, 305-309.

Castilho, P., Pinto-Gouveia, J. and Duarte, J. (2015). Evaluating the multifactor structure of the long and short versions of the Self-Compassion Scale in a clinical sample. Journal of Clinical Psychology, $71,856-870$.

Cloitre, M., Roberts, N. P., Bisson, J. I. and Brewin, C. R. (2017). The International Trauma Questionnaire (ITQ). Unpublished measure. 
Dutton, M. A., Bermudez, D., Matas, A., Majid, H. and Myers, N. L. (2013). Mindfulness-based stress reduction for low-income, predominantly African American women with PTSD and a history of intimate partner violence. Cognitive and Behavioral Practice, 20, 23-32.

Gilbert, P. (2010). Compassion focused therapy: Distinctive features. London: Routledge

Gilbert, P. and Irons, C. (2014). Compassion Focused Therapy (CFT). In W. Dryden and A. Reeves (eds), Handbook of Individual Therapy (sixth edition). Sage: London

Gray, M. J., Litz, B. T., Wang, J. and Lombardo, T. W. (2004). Psychometric properties of the Life Events Checklist. Assessment, 11, 330-341.

Hayes, S. C., Strosahl, K. and Wilson, K. G. (1999). Acceptance and Commitment Therapy: An Experiential Approach to Behavior Change. New York: Guilford Press.

Johnson, E. A. and O'Brien, K. A. (2013). Self-compassion soothes the savage ego-threat system: effects on negative affect, shame, rumination, and depressive symptoms. Journal of Social and Clinical Psychology, 32, 939-963.

Kabat-Zinn, J. (1990). Full Catastrophe Living: Using the Wisdom of your Body and Mind to Face Stress, Pain, and Illness. New York: Delacorte.

Karatzias, T., Shevlin, M., Fyvie, C., Hyland, P., Efthymiadou, E., Wilson, D. et al. (2016). An initial psychometric assessment of an ICD-11 based measure of PTSD and complex PTSD (ICD-TQ): evidence of construct validity. Journal of Anxiety Disorders, 44, 73-79.

Karatzias, T., Shevlin, M., Fyvie, C., Hyland, P., Efthymiadou, E., Wilson, D. et al. (2017). Evidence of distinct profiles of posttraumatic stress disorder (PTSD) and complex posttraumatic stress disorder (CPTSD) based on the new ICD-11 Trauma Questionnaire (ICD-TQ). Journal of Affective Disorders, 207, 181-187.

Kearney, D. J., McDermott, K., Malte, C., Martinez, M. and Simpson, T. L. (2012). Association of participation in a mindfulness program with measures of PTSD, depression and quality of life in a veteran sample. Journal of Clinical Psychology, 68, 101-116.

Krieger, T., Altenstein, D., Baettig, I., Doerig, N. and Holtforth, M. G. (2013). Self-compassion in depression: associations with depressive symptoms, rumination, and avoidance in depressed outpatients. Behavior Therapy, 44, 501-513.

Lee, D. (2016). Compassion Focused Therapy: Is Compassion an Antidote to Shame and an Effective Treatment of Complex PTSD? Master Clinician Workshop at the International Society of Traumatic Stress Studies Annual Meeting, Dallas.

Lee, D. and James, S. (2012). The Compassionate Mind Approach to Recovering from Trauma using Compassion Focused Therapy. London: Constable and Robinson.

Linehan, M. M. (1993). Diagnosis and treatment of mental disorders. Cognitive-behavioral treatment of borderline personality disorder. New York: Guilford Press.

MacBeth, A. and Gumley, A. (2012). Exploring compassion: a meta-analysis of the association between self-compassion and psychopathology. Clinical Psychology Review, 32, 545-552.

Maercker, A., Brewin, C. R., Bryant, R. A., Cloitre, M., Reed, G. M., van Ommeren, M. and Saxena, S. (2013). Proposals for mental disorders specifically associated with stress in the ICD-11. Lancet, 381, 1683-1685. doi: 10.1016/S0140-6736

Maheux, A. and Price, M. (2015) Investigation of the relation between PTSD symptoms and selfcompassion: comparison across DSM IV and DSM 5 PTSD symptom clusters. Self and Identity, 14, 627-637.

Neff, K. D. (2003a). Self-compassion: an alternative conceptualization of a healthy attitude toward oneself. Self and Identity, 2, 85-102.

Neff, K. D. (2003b). The development and validation of a scale to measure self-compassion. Self and Identity, 2, 223-250.

Neff, K. D. (2011). Self-compassion, self-esteem, and well-being. Social and Personality Psychology Compass, 5, 1-12. 
Scoglio, A. A, Rudat, D. A., Garvert, D., Jarmolowski, M., Jackson, C. and Herman, J. L. (2015). Self-compassion and responses to trauma: the role of emotion regulation. Journal of Interpersonal Violence. doi: 10.1177/0886260515622296

Segal, Z. V., Williams, J. M. G. and Teasdale, J. D. (2002). Mindfulness-based cognitive therapy for depression: A new approach to preventing relapse. New York: Guilford Press.

Tabachnick, B. G. and Fidell, L. S. (2001). Using Multivariate Statistics. New York: Harper and Collins.

Thompson, B. L. and Waltz, J. (2008). Self-compassion and PTSD symptom severity. Journal of Traumatic Stress, 21, 556-558. 